\title{
Long-Term Mechanical Properties of Smart Cable Based on FBG Desensitized Encapsulation Sensors
}

\author{
Sheng $\mathrm{LI}^{1 *}$ and Min $\mathrm{ZHOU}^{2}$ \\ ${ }^{1}$ National Engineering Laboratory for Fiber Optic Sensing Technology, Wuhan University of Technology, Wuhan, \\ 430070, China \\ ${ }^{2}$ Wuhan WUTOS Limited Company, Wuhan, 430223, China \\ *Corresponding author: Sheng LI_ E-mail: lisheng@whut.edu.cn
}

\begin{abstract}
In order to ensure the testing range and long-term reliability of the fiber Bragg grating (FBG) used for the smart cable, a smart cable embedded with FBG strain sensors based on the desensitized encapsulation structure was designed. For a smart cable specimen, the fatigue loading experiments with the cycle from 100 thousands to 2 million and 0.95 times nominal breaking cable force $\left(P_{b}\right)$ were carried out, which tested the long-serving effects of the smart cable. The test results of the static tension loading and unloading during the stepwise fatigue cycle process showed that the encapsulated FBG strain sensors had the good linearity and repeatability. Also all sensors survived after 2 million times fatigue cycle. $0.95 P_{b}$ static tension test showed that the encapsulated FBG strain sensors embedded inside the cable reached $4.5 \%$ testing accuracy in the $0.86 P_{b}$ working range. After $0.95 P_{b}$ static tension test, the dissection test was carried out by breaking the force tension. The results showed that the appearances of the encapsulated sensors were good, and the design structures were not changed and damaged.
\end{abstract}

Keywords: Smart cable, fatigue experiment, fiber Bragg grating, force monitoring, bridge engineering

Citation: Sheng LI and Min ZHOU, "Long-Term Mechanical Properties of Smart Cable Based on FBG Desensitized Encapsulation Sensors," Photonic Sensors, 2014, 4(3): 236-241.

\section{Introduction}

Forming a smart cable by embedding optical fiber sensors inside a cable in the fabricating stage has been widely researched. The fiber Bragg grating (FBG) sensor $[1,2]$ is a mainly sensing means for the smart cable [3-5]. Also there are some studies on the smart cable by the Brillouin sensing technique [6]. The difficulties in the FBG-based smart cable are to increase the reliable strain range which requires for $5000 \mu \varepsilon$ [7], close to the rupture limit strain of the naked FBG [8], and the compatibility process requirements should conform to the conventional cable manufacturing processes. The existing research on the smart cable mainly focuses on the short-term property performances by the static loading test and is lack of the consideration of the long-term properties.

In order to meet the large strain working range of the cable in the over tensioning stage and the maximum combined load in the operational stage, an FBG desensitized encapsulation sensor was studied for solving the range problem effectively. In addition, based on Chinese code [9] about the fatigue requirements for the steel wires of the cable, 2 million times fatigue cycle experiment and 
0.95 times nominal breaking cable force $\left(P_{b}\right)$ loading experiment were carried out for a specimen smart cable.

\section{Structure of the smart cable}

\subsection{Sensing principle}

For the traditional single steel tube encapsulation structure, the cooperative deformation between the steel tube and the testing object occurs prior to the deformation of the FBG driven by the encapsulated steel tube. So when subjected to the large stress, the strain transfer process usually cannot totally finish due to the reason that sensor pedestals fastened to the steel wire may break off the encapsulation structure.

So as shown in Fig. 1, the FBG is mainly encapsulated by three-segment steel tubes. The boundary of the steel tube in the middle only fastens one side, and the other side is completely free. The strain transfer pathway of the encapsulation structure can be expressed as: steel wire of the cable $\rightarrow$ adhesive $\rightarrow$ pedestal $\rightarrow$ fiber optical fixed-point at the ends $\rightarrow$ FBG. In essence, the middle steel tube only protects the FBG from the lateral load and the impact of the shear force. And after the transfer through the pedestal, the FBG directly bears the tensile strain. Set that $L_{s}$ and $L_{\mathrm{FBG}}$ delegate the distance between two pedestals and the distance between two optical fiber fixing points, respectively. When the steel wire tensile deformation occurs, the deformation coordination relationship between the steel wire strain $\varepsilon$ and the optical fiber strain $\varepsilon_{f}$ can be expressed as

$$
\varepsilon L_{s}=\varepsilon_{f} L_{\mathrm{FBG}} .
$$

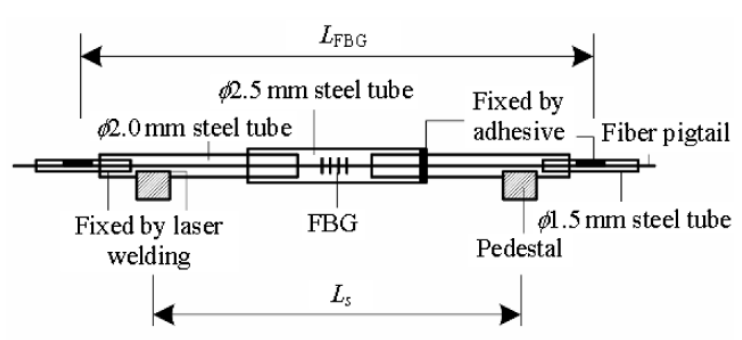

Fig. 1 Layout of FBG strain sensors.
Thus, the strain sensor sensitivity can be adjusted by setting different ratios of $L_{s}$ and $L_{\mathrm{FBG}}$. When $L_{\mathrm{FBG}}>L_{s}$, the strain sensitivity of the FBG wavelength drift decreases compared to the steel wire strain, which achieves the sensor desensitization. Choosing the proper ratio of $L_{s}$ and $L_{\mathrm{FBG}}$ can ensure the reliable test range requirement of the FBG sensor under the service load of the cable.

\subsection{Desensitized test}

Three specimens of the proposed FBG sensors with different ratios of $L_{s}$ and $L_{\mathrm{FBG}}$ were used for the tension test based on the single steel wire in the laboratory. As shown in Table 1, the experimental results of the three specimens showed that the actual values calculated from the tension test were a little bigger than the theoretical values due to the transmission force influence of the adhesive between the pedestals and testing steel wire. However, three specimens all achieved the aim of desensitization. The actual strain sensitivity coefficients were all less than the theoretical values of the naked FBG, namely less than $1 \mathrm{pm} / \mu \varepsilon$. Taking the best desensitized effect of the 3rd length for example as Fig. 2, three specimens all had the good repeatability. And the fitting linear correlations all exceeded 0.99 .

Table 1 Strain sensitivity coefficient of different structure dimension sensors (unit: $\mathrm{pm} / \mu \varepsilon$ ).

\begin{tabular}{cccc}
\hline Strain sensitivity coefficient & 1st specimen & 2nd specimen & 3rd specimen \\
\hline Theoritical value & 0.679 & 0.593 & 0.519 \\
Actual value & 0.724 & 0.654 & 0.559 \\
\hline
\end{tabular}

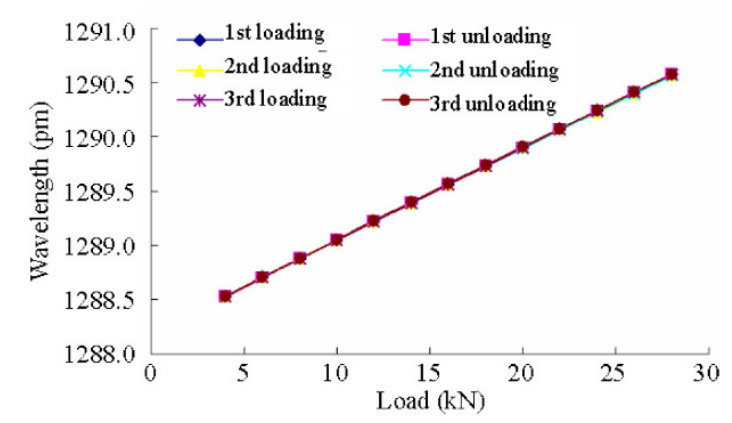

Fig. 2 Load versus wavelength relationship curves of the 3 rd specimen. 


\subsection{Fabrication scheme}

The fabrication scheme of the smart cable mainly contained eight steps, which were depicted in Fig. 3. To avoid the effect of the high temperature epoxy stuffing on the fiber, the fifth step was arranged after the fourth step. In the fifth step, the FBG sensors were fastened to the periphery steel wires of the cable in the connection pipe region. The layout of the FBG sensors in the smart cable is shown in Fig. 4. The reserved channels in the first step provided the through space for steel tubes which gave the protective measure to the output optical fiber. The calibration test of the smart cable was the last step, which utilized the over tensioning test procedure for every cable.

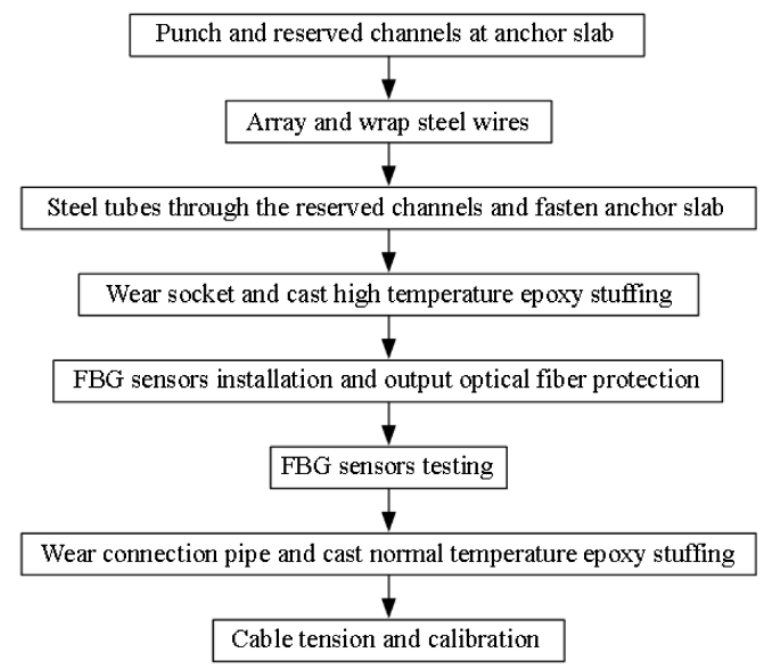

Fig. 3 Fabrication schemes of smart cables with FBG sensors.

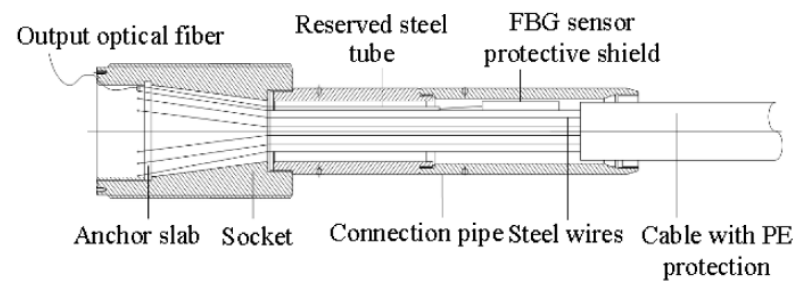

Fig. 4 Layout of the FBG sensors in one smart cable.

\section{Mechanical property testing}

\subsection{Object and purpose}

As shown in Fig. 5, a 3.5-meter-length smart cable with the $85 \phi 7$ smart cable was fabricated with the help of Jiangsu FASTEN Group Company (steel wire nominal strength $\sigma_{b}=1670 \mathrm{MP}_{\mathrm{a}}$ ). The smart cable embedded five designed FBG strain sensors and one FBG temperature sensor used for the temperature compensation. The main purpose of the test was to check the long-term mechanical properties of the smart cable based on Chinese code about fatigue requirements for the steel wires of the cable.
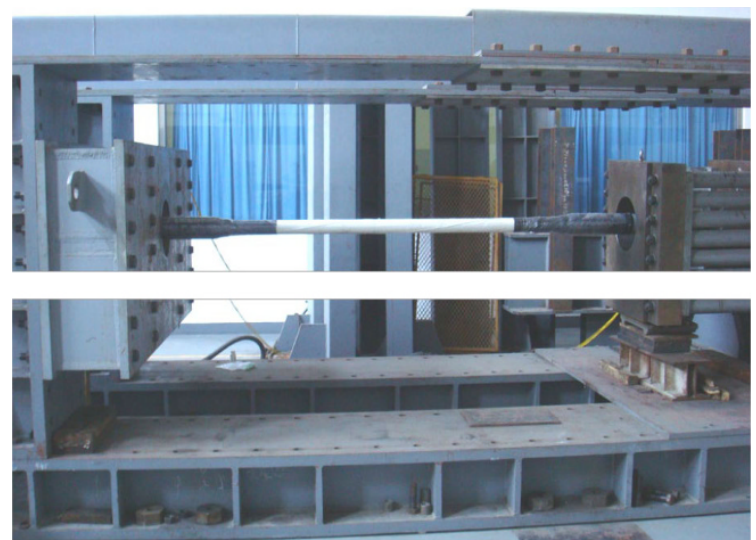

Fig. 5 Smart cable test specimen.

\subsection{Methods and steps}

The whole test process is shown in Fig. 6, and before the fatigue test, the force sensitivity coefficient reference was established by the two-cycle loading and unloading tension test with $300 \mathrm{kN}$ load interval from $300 \mathrm{kN}$ to $2100 \mathrm{kN}$. Due to different actual installation locations, the force sensitivities to the steel wires strain of five sensors were different, as shown in Table 2. However, both the linear correlation and repeatability of all sensors were good. Figure 7 presents the load versus wavelength relationship curve of $1 \#$ sensor. According to the code, the upper limit of the fatigue stress was set at $668 \mathrm{MPa}\left(0.40 \sigma_{b}\right)$, and the stress range $\Delta \sigma$ was set at $200 \mathrm{MPa}$. The static loading tension tests with $0.4 P_{b}$ range were carried out after every stage fatigue cycle experiment adopted by sine wave load with the 2-Hz frequency, which used for tracking the stability of the smart cable output signal and analyzing the force sensitive coefficient time varying trend. In addition, after 2 million times 
fatigue cycle, $0.95 P_{b}$ range static tension and breaking force tension tests were carried out for checking the maximum working range of the smart cable and the appearance state of the designed sensors inside the cable.

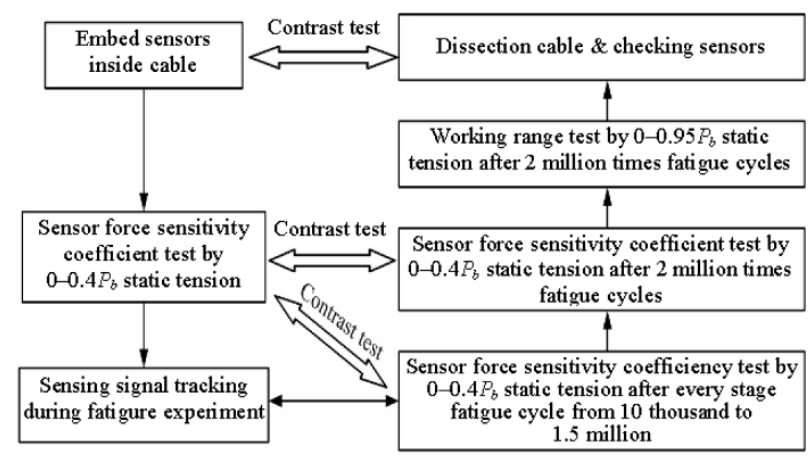

Fig. 6 Testing process for smart cable.

Table 2 Force sensitivity coefficient reference of each sensor (unit: $\mathrm{kN} / \mathrm{pm}$ ).

\begin{tabular}{cccccc}
\hline Sensor & $1 \#$ & $2 \#$ & $3 \#$ & $4 \#$ & $5 \#$ \\
\hline Force sensitivity coefficient & 1.947 & 1.363 & 1.174 & 1.622 & 1.656 \\
\hline
\end{tabular}

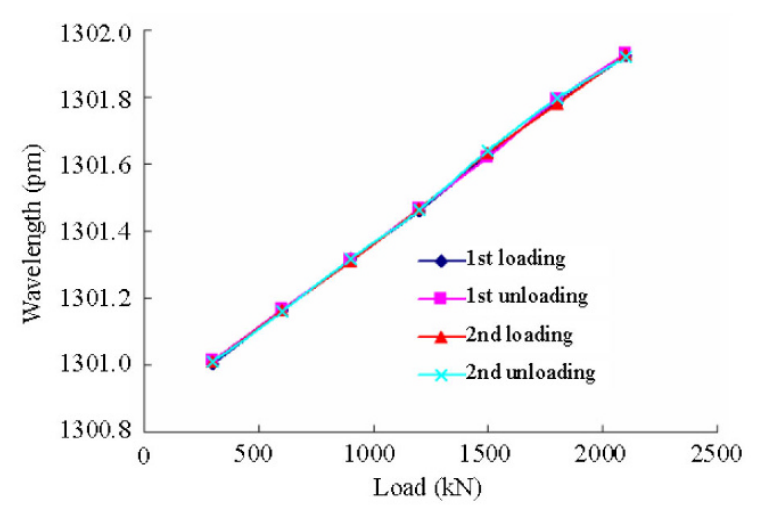

Fig. 7 Load versus wavelength relationship curve of $1 \#$ sensor.

\subsection{Results and analysis}

All designed sensors survived after 2 million times fatigue cycle. The force of the smart cable was delegated by the cable force output average of five designed FBG strain sensors which considered the temperature compensation and took the static tension strain sensitivity coefficients before the fatigue experiment as the calculation basis. Before and after 2 million times fatigue, the results of every step output cable force under $0.4 P_{b}$ range static loading tension test were shown in Tables 3 and 4. The maximum force errors between the loading and testing did not beyond $50 \mathrm{kN}$ and $200 \mathrm{kN}$ before and after the fatigue experiment.

Table 3 Force of the smart cable before fatigue (unit: $\mathrm{kN}$ ).

\begin{tabular}{ccccc}
\hline Case & 1st loading & 1st unloading & 2nd loading & 2nd unloading \\
\hline 600 & 620.1 & 599.5 & 648.6 & 590.6 \\
900 & 938.2 & 889.3 & 948.9 & 887.0 \\
1200 & 1228.1 & 1175.1 & 1240.6 & 1167.7 \\
1500 & 1517.6 & 1466.2 & 1524.8 & 1478.0 \\
1800 & 1804.5 & 1772.9 & 1814.1 & 1777.8 \\
2100 & 2109.3 & 2109.3 & 2107.5 & 2107.5 \\
\hline
\end{tabular}

Table 4 Force of the smart cable after fatigue (unit: $\mathrm{kN}$ ).

\begin{tabular}{ccccc}
\hline Case & 1st loading & 1st unloading & 2nd loading & 2nd unloading \\
\hline 600 & 465.9 & 448.2 & 458.2 & 454.1 \\
900 & 842.1 & 826.4 & 837.5 & 806.7 \\
1200 & 1210.6 & 1191.7 & 1214.9 & 1186.9 \\
1500 & 1589.6 & 1559.5 & 1586.6 & 1564.5 \\
1800 & 1923.0 & 1941.9 & 1935.0 & 1927.1 \\
2100 & 2276.7 & 2298.1 & 2285.6 & 2299.7 \\
\hline
\end{tabular}

The force errors were caused by the change in the force sensitivity coefficients during the fatigue cycle process. Figure 8 shows the development trend of the sensor force sensitive coefficients calculated from the relationship between the testing load and sensor wavelength.

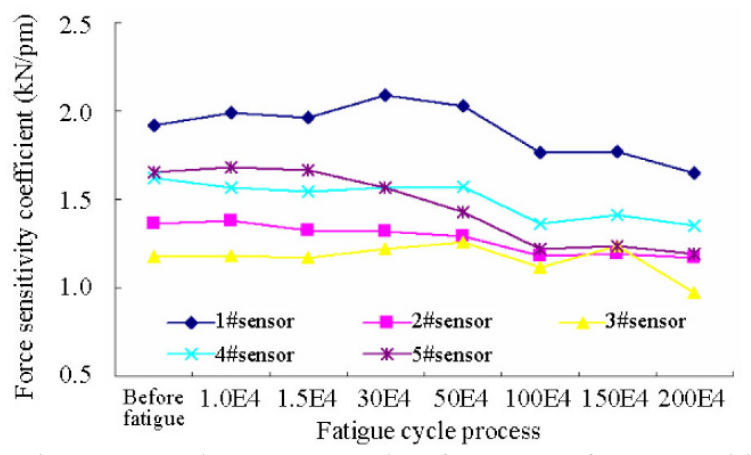

Fig. 8 Development trend of sensor force sensitive coefficients.

After 2 million times fatigue cycle, $0.95 P_{b}$ static tension test was carried out. Table 5 shows the maximum working range of five designed FBG 
sensors. During the static tension process, $1 \#-3 \#$ sensors all survived, while $4 \#$ sensor was failure at $0.8627 P_{b}$ (as shown in Fig. 9) and 5\# sensor was failure at $0.89 P_{b}$. So the maximum working range of the smart cable could be set at $0.86 P_{b}$. The cable force testing accuracy change during the fatigue cycle process is shown in Fig. 10 based on (2). With an increase in the fatigue cycle, the testing accuracy decreased gradually. After 2 million times fatigue cycle, the accuracy still reached $4.5 \%$.

Accuracy $=\mid$ m axim um error $\mid /$ range $\times 100 \%$. (2)

Table 5 Sensor testing range.

\begin{tabular}{cccccc}
\hline Sensor No. & $1 \#$ & $2 \#$ & $3 \#$ & $4 \#$ & $5 \#$ \\
\hline$P_{b}(\%)$ & $\geqslant 0.95$ & $\geqslant 0.95$ & $\geqslant 0.95$ & 0.86 & 0.89 \\
\hline
\end{tabular}

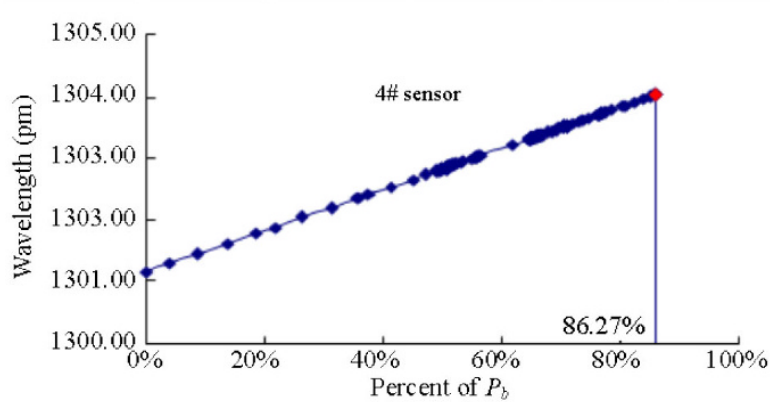

Fig. 9 Wavelength drifts of $4 \#$ sensor during the tension loading process.

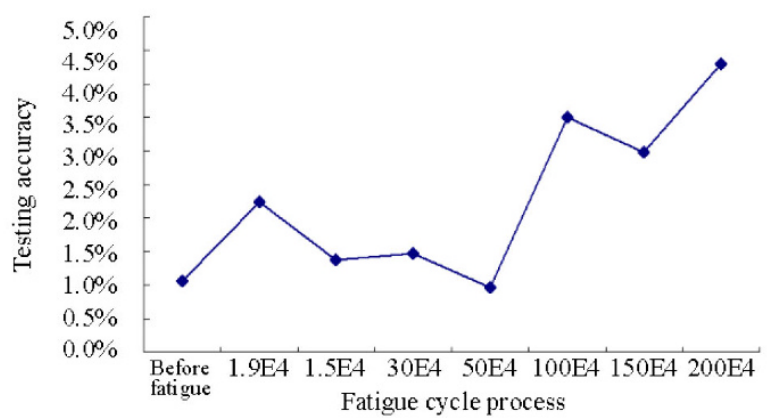

Fig. 10 Cable force testing accuracy change during the fatigue cycle process.

There was no break of the smart cable after $0.95 P_{b}$ static tension test. The appearance inspection results after unloading showed that the anchorage and cable were both in good condition. According to the breaking force tension, the dissection for the smart cable was finished. As shown in Fig. 11, by appearance checking, the encapsulated sensors under the protective shields were good, and the designed structures were not changed and damaged compared to the installation stage.

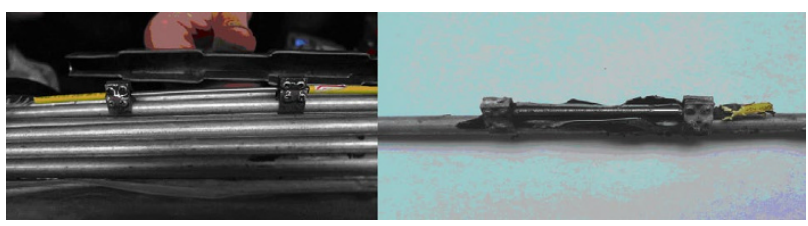

Fig. 11 Sensor appearance contrast between the installation and dissection states.

\section{Conclusions}

The FBG-based smart cable is a meaningful smart structure. The desensitized encapsulation solves the large strain measurement problem when using the FBG sensor. The proposed cable fabricating scheme can ensure a high survival rate for the sensor. The fatigue experiment results showed that after long-term loading the smart cable had $4.5 \%$ testing accuracy in the $0.86 P_{b}$ working range. The breaking force tension and dissection experiments verified the long-term effectiveness of the designed sensor and installation structure. The proposed study plan based on fatigue code requirements for the steel wires of cable can provide a reference for studying the long-term mechanical properties of other type of the smart cable structure.

\section{Acknowledgment}

The research work reported in this paper was jointly supported by the National Engineering Laboratory for Fiber Optic Sensing Technology, Wuhan University of Technology, China and FASTEN Group Company. Thanks to the support of Wuhan City Building Research Funds (201310), the Fundamental Research Funds for the Central Universities (WUT: 2014-IV-090), and the National Natural Science Foundation of China (Major Program: 61290310).

Open Access This article is distributed under the terms of the Creative Commons Attribution License which permits any use, distribution, and reproduction in any medium, provided the original author(s) and source are credited. 


\section{References}

[1] T. Wang, Z. Yuan, Y. Gong, Y. Wu, Y. Rao, L. Wei, et al., "Fiber Bragg grating strain sensors for marine engineering," Photonic Sensors, 2013, 3(3): 267-271.

[2] J. Wang, T. Liu, G. Song, H. Xie, L. Li, X. Deng, et al., "Fiber Bragg grating (FBG) sensors used in coal mines," Photonic Sensors, 2014, 2(4): 120-124.

[3] T. Li, C. Li, and H. Liu, "Stress measurement at cable and anchor end by using fiber Bragg grating," China Journal of Highway and Transport, 2012, 25(5): 134-138.

[4] J. Wu, W. Chen, P. Zhang, L. Liu, and L. Liu, "Smart cable technology based on fiber Bragg grating strain sensing in cable anchorage zone," Chinese Journal of Lasers, 2010, 37(6): 1510-1515.

[5] Z. Zhou, Z. Zhang, N. Deng, X. Zhao, and D. Li, "Application of FRP-OFBG sensors on bridge cables," in Proc. SPIE (Smart Structures \&
Material/NDE Joint Conference: Sensors and Smart Structures for Civil, Mechanical and Aerospace System), vol. 5765, pp. 668-677, 2005.

[6] Z. Zhou, J. He, H. Jia, and J. Ou, "Full scale Brillouin sensing technique based smart parallel wire stay cable," Journal of Optoelectronics Laser, 2009, 20(6): 766-770.

[7] S. LI and D. Jiang, "Structural large strain monitoring based on FBG sensor," in Symposium on Photonics and Optoelectronics (SOPO2009), Wuhan, China, August 14-16, 2009.

[8] T. Liu, J. Jiang, and X. Li, “Application of fiber Bragg grating sensor on measurement of tensile stress in a seven-wire prestressed steel strand," Journal of Optoelectronics Laser, 2005, 16(10): 1235-1238.

[9] Chongqing communications research \& design institute, Technical conditions for hot-extruding PE protection high strength wire cable of cable-stayed bridge (GBT18365-2001). Beijing: China Standards Press, 2001. 